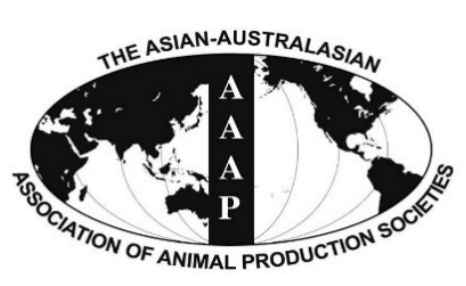

Open Access

Asian Australas. J. Anim. Sci.

Vol. 29, No. 4 : 471-478 April 2016

http://dx.doi.org/10.5713/ajas.15.0915

www.ajas.info

pISSN 1011-2367 elSSN 1976-5517

\title{
Genetic Diversity Analysis of South and East Asian Duck Populations Using Highly Polymorphic Microsatellite Markers
}

\author{
Dongwon Seo, Md. Shamsul Alam Bhuiyan', Hasina Sultana, Jung Min Heo, and Jun Heon Lee* \\ Division of Animal and Dairy Science, Chungnam National University, Daejeon 34134, Korea
}

\begin{abstract}
Native duck populations have lower productivity, and have not been developed as much as commercials duck breeds. However, native ducks have more importance in terms of genetic diversity and potentially valuable economic traits. For this reason, population discriminable genetic markers are needed for conservation and development of native ducks. In this study, 24 highly polymorphic microsatellite (MS) markers were investigated using commercial ducks and native East and South Asian ducks. The average polymorphic information content (PIC) value for all MS markers was 0.584 , indicating high discrimination power. All populations were discriminated using 14 highly polymorphic MS markers by genetic distance and phylogenetic analysis. The results indicated that there were close genetic relationships among populations. In the structure analysis, East Asian ducks shared more haplotypes with commercial ducks than South Asian ducks, and they had more independent haplotypes than others did. These results will provide useful information for genetic diversity studies in ducks and for the development of duck traceability systems in the market. (Key Words: Discrimination, Duck, Genetic Diversity, Microsatellite Marker)
\end{abstract}

\section{INTRODUCTION}

Native breeds in rural areas are important genetic resources for maintaining genetic diversity and introducing potentially valuable economic characteristics. However, because of economic development caused by rapid industrialization over the past half-century, commercial breeds have rapidly increased in comparison with native breeds owing to the need for efficient commercial production. Faster growth rates and higher productivity of commercial breeds have been continuously developed. Conversely, productivity of native breeds has declined, populations have decreased in size, and some breeds are extinct, all of which indicate a decline in genetic diversity. However, need for the conservation of biodiversity of native livestock species has recently begun to be recognized (Fulton and Delany, 2003).

Duck meat is recognized as a healthy food in Asian

\footnotetext{
* Corresponding Author: Jun Heon Lee. Tel: +82-42-821-5779, Fax: +82-42-825-9754, E-mail: junheon@cnu.ac.kr

${ }^{1}$ Department of Animal Breeding and Genetics, Bangladesh Agricultural University, Mymensingh 2202, Bangladesh.

Submitted Nov. 11, 2015; Revised Dec. 24, 2015; Accepted Jan. 14, 2016
}

countries. In particular, duck meat consumption in Korea has rapidly increased over the last decade. Duck meat is rich in unsaturated fatty acids and consumer preference for healthy meat is now three times greater than that during the last decade (MIFFAF, 2013). Southeast Asian countries have conserved various native duck species because the center of duck meat consumption in Southeast Asia, including Korea, is located in a migratory bird flyway (Kraus et al., 2011). However, the commercial use of native ducks is less than that of the Peking breed, a commercial duck, which has a higher growth rate and is more productive (Kim et al., 2012). Native breeds need to be protected for biodiversity conservation and development of economically important traits, which would also be valuable ecologically and to breed survival. Therefore, development of breed-discriminating genetic markers and conservation strategies is important for breed conservation and trait development. In addition, duck breed classification analysis can provide additional information for preventive veterinary inspections for avian influenza (AI) viruses (Groepper et al., 2014; Ramey et al., 2014).

The origin of domestic ducks may be from the Mallard (Anas platyrhynchos) and Spot-billed Duck (Anas 
poecilorhyncha). They ae both migratory birds, but may settle in areas with good food and habitat. They also have characteristics that allow them to be easily domesticated. In addition, Mallards may cross with Spot-billed Duck, and the hybrids appear in natural habitats (Jin et al., 2014). Most domestic ducks are difficult to classify using genetic makers because they share these wild duck ancestors.

Until recently, various types of molecular markers using mtDNA sequence information, microsatellite (MS) genotyping, and single nucleotide polymorphisms (SNPs) to increase industrial livestock value have only been applied in species such as cattle, pigs, and chickens (Dalvit et al., 2007; Tadano et al., 2007). Genetic markers for duck breed discrimination have primarily been developed from Chinese ducks rather than those from other Asian countries. Many duck breeds and genetic linkage maps have been reported (Maak et al., 2003; Yinhua et al., 2005; Huang et al., 2006). Some wild duck classification studies have used cytochrome oxidase I (COI) and D-loop control region sequence phylogeny analysis, but there were difficulties in discriminating domestic ducks that shared alleles from wild Mallards and Spot-billed Duck ancestors (Jin et al., 2012). Otherwise, wild ducks were discriminated well using mtDNA sequence information (Jin et al., 2014). MS marker studies also reported various MS marker polymorphisms (Maak et al., 2003; Yinhua et al., 2005; Huang et al., 2006), but phylogenetic or discrimination analysis resulted in few groups using microsatellite genotype information (Seo et al., 2015). MS markers are useful tools for livestock traceability systems in addition to use in human forensic science. MS markers have enough discriminating power because of higher polymorphic than other genetic markers. MS markers have 2 to $5 \mathrm{bp}$ of simple sequence repeats and genotype results can be confirmed when compared to amplicon size with fluorescence dye (Groenen et al., 1998). Recently, many studies have applied high-density (HD) SNP markers because of their large-scale platform and the large amount of genetic information for cattle, pig, and chicken studies (Ramos et al., 2009; Stothard et al., 2011; Kranis et al., 2013). However, HD-SNP analysis is limited in duck studies. Although large amounts of SNPs have been reported (Kraus et al., 2011), to our knowledge, no HD-SNP platforms have been developed yet. Therefore, the objective of this study was to locate highly polymorphic duck MS marker combinations for confirmation of genetic diversity and discrimination of domestic duck populations from South and East Asia.

\section{MATERIALS AND METHODS}

\section{Samples and DNA extraction}

The native Asian duck samples were provided by the National Institute of Animal Science (NIAS) in Korea and Bangladesh Agricultural University (BAU) in Bangladesh.
These native Asian duck populations consisted of three different Korean native duck (KND) populations (KND_Y: Yong-in white KND [ $\mathrm{n}=72]$; KND_J: Jang-sung white KND $[\mathrm{n}=58]$; KND_C: Jang-sung colored KND [ $\mathrm{n}=9]$ ) that are primarily classified by feather color (white versus colored). Four different Bangladeshi native duck (Ba) populations were included in this study (BaL: Bangladeshi Local duck [n $=13]$; BaJ: Bangladeshi Jinding duck $[\mathrm{n}=15]$; BaW: Bangladeshi white $[\mathrm{n}=20]$; BaB; Bangladeshi black $[\mathrm{n}=$ 36]). All possible precautions were taken during sampling from unrelated individuals to prevent cross contamination. The genomic DNA of blood samples was extracted using PrimePrep Genomic DNA Isolation Kits for Blood (GenetBio, Daejeon, Korea). The quality and concentration of extracted DNA was determined by electrophoresis using $0.8 \%$ agarose gel, as well as with a spectrophotometer using the NanoDrop 2000C (Thermo Scientific, Waltham, MA, USA).

\section{Marker selection and genotyping}

From the previous study by Seo et al. (2015), we selected 24 polymorphic MS markers for Asian duck discrimination analysis. All MS markers were modified for four types of fluorescence dye (FAM, VIC, NED, and PET) in forward primers. For this analysis, polymerase chain reaction (PCR) amplification was performed in an initial denaturation at $95^{\circ} \mathrm{C}$ for $10 \mathrm{~min}$ followed by 31 cycles of $30 \mathrm{~s}$ at $95^{\circ} \mathrm{C}, 30 \mathrm{~s}$ at $63^{\circ} \mathrm{C}, 30 \mathrm{~s}$ at $72^{\circ} \mathrm{C}$, and a final extension at $72^{\circ} \mathrm{C}$ for 10 min using the $\mathrm{C} 1000$ Thermal Cycler (Bio-Rad, USA). A total of $20 \mu \mathrm{L}$ reaction volume of PCR reagent contained 50 ng of gDNA, 2× Multi HS Prime Taq Premix (GenetBio, Korea), 8 pico mole of each forward and reverse primer, and was adjusted using distilled water. The genotyping mixture contained $1 \mu \mathrm{L}$ of PCR product, $10 \mu \mathrm{L}$ of Hi-Di formamide (Applied Biosystems, Waltham, MA, USA), and $0.1 \mu \mathrm{L}$ of the GeneScan-500LIZ size standard (Applied Biosystems, USA). Fragment analysis was performed using the Genetic Analyzer 3130xl (Applied Biosystems, USA) and the repeat variation results were obtained using Genemapper ver. 4.1 (Applied Biosystems, USA).

\section{Statistical analysis}

The obtained size represented binary values and the number of alleles $(\mathrm{N})$, observed heterozygosity (Ho), expected heterozygosity $(\mathrm{He})$, and polymorphic information content (PIC) values were calculated using the Cervus ver 3.0.7 program (Marshall et al., 1998). The genetic distances for phylogenetic analysis (Neighbor-Joining tree; NJ tree) were calculated using the PowerMarker ver. 3.25 program (Liu and Muse, 2005). Structure analysis for genetic inference of haplotype distribution was performed using the Structure ver. 2.3.4 program (Pritchard et al., 2000). The probability of identity $(P I)$ values in random, half-sib, and 
full-sib populations were calculated using the API-CALC ver. 1.0 program (Ayres and Overall, 2004).

\section{RESULTS AND DISCUSSION}

\section{Polymorphism of microsatellite markers}

Polymorphisms are important components for discrimination of populations and individuals. MS marker genotypes of the experimental populations were all polymorphic. The $24 \mathrm{MS}$ markers had an average of 9.38 alleles, $0.492 \mathrm{Ho}, 0.623 \mathrm{He}$, and 0.584 PIC. The highest polymorphic marker, CAUD040, had the greatest number of alleles (29) and highest expected He (0.938) and PIC (0.932) values (Table 1). In addition, the lowest polymorphic marker, AMU52, had the minimum number of alleles (three) and lowest He (0.037) and PIC (0.036) values (Table 1). Among the 24 selected MS markers, the number of alleles varied from three to 29 , Ho was 0.029 to 0.871 , He was 0.037 to 0.938 , and PIC was 0.036 to 0.932 (Table 1 ).

The number of alleles and He and PIC values can provide important information for the discrimination of individuals

Table 1. Polymorphism information for microsatellite markers used in this study

\begin{tabular}{lccccc}
\hline Locus & $\mathrm{k}$ & $\mathrm{N}$ & $\mathrm{Ho}$ & $\mathrm{He}$ & $\mathrm{PIC}$ \\
\hline *CAUD111 & $\mathbf{7}$ & $\mathbf{2 4 3}$ & $\mathbf{0 . 6 2 6}$ & $\mathbf{0 . 7 3 1}$ & $\mathbf{0 . 6 8 6}$ \\
CAUD127 & 7 & 243 & 0.206 & 0.284 & 0.257 \\
CAUD132 & 4 & 243 & 0.222 & 0.355 & 0.297 \\
AMU52 & 3 & 243 & 0.029 & 0.037 & 0.036 \\
CAUD044 & 11 & 243 & 0.531 & 0.635 & 0.597 \\
*AMU68 & $\mathbf{8}$ & $\mathbf{2 4 3}$ & $\mathbf{0 . 6 0 5}$ & $\mathbf{0 . 6 8 8}$ & $\mathbf{0 . 6 5 5}$ \\
CAUD009 & 5 & 243 & 0.041 & 0.225 & 0.207 \\
*APH04 & $\mathbf{1 0}$ & $\mathbf{2 4 3}$ & $\mathbf{0 . 5 6 4}$ & $\mathbf{0 . 7 6}$ & $\mathbf{0 . 7 3 2}$ \\
AMU123 & 6 & 243 & 0.395 & 0.56 & 0.507 \\
*APH08 & $\mathbf{5}$ & $\mathbf{2 4 3}$ & $\mathbf{0 . 6 1 7}$ & $\mathbf{0 . 7 1 7}$ & $\mathbf{0 . 6 7 1}$ \\
*CAUD005 & $\mathbf{9}$ & $\mathbf{2 4 3}$ & $\mathbf{0 . 7 0 8}$ & $\mathbf{0 . 7 6 6}$ & $\mathbf{0 . 7 2 8}$ \\
CAUD128 & 4 & 243 & 0.395 & 0.396 & 0.342 \\
*AMU3 & $\mathbf{4}$ & $\mathbf{2 3 9}$ & $\mathbf{0 . 4 6 4}$ & $\mathbf{0 . 6 4 3}$ & $\mathbf{0 . 5 9}$ \\
*CAUD069 & $\mathbf{2 0}$ & $\mathbf{2 2 6}$ & $\mathbf{0 . 5 1 8}$ & $\mathbf{0 . 8 9 3}$ & $\mathbf{0 . 8 8 3}$ \\
*CAUD086 & $\mathbf{1 1}$ & $\mathbf{2 4 3}$ & $\mathbf{0 . 6 9 5}$ & $\mathbf{0 . 7 6 5}$ & $\mathbf{0 . 7 3}$ \\
*APH20 & $\mathbf{5}$ & $\mathbf{2 4 3}$ & $\mathbf{0 . 6 1 7}$ & $\mathbf{0 . 7 2 1}$ & $\mathbf{0 . 6 7 4}$ \\
*CAUD066 & $\mathbf{8}$ & $\mathbf{2 4 0}$ & $\mathbf{0 . 4 8 3}$ & $\mathbf{0 . 7 2 8}$ & $\mathbf{0 . 6 8 1}$ \\
APH24 & 6 & 240 & 0.371 & 0.543 & 0.502 \\
*CAUD039 & $\mathbf{8}$ & $\mathbf{2 4 3}$ & $\mathbf{0 . 5 2 7}$ & $\mathbf{0 . 7 4 8}$ & $\mathbf{0 . 7 1 2}$ \\
*CAUD040 & $\mathbf{2 9}$ & $\mathbf{2 4 0}$ & $\mathbf{0 . 8 7 1}$ & $\mathbf{0 . 9 3 8}$ & $\mathbf{0 . 9 3 2}$ \\
CAUD011 & 5 & 243 & 0.519 & 0.573 & 0.492 \\
CAUD031 & 10 & 236 & 0.466 & 0.612 & 0.533 \\
*CAUD035 & $\mathbf{1 3}$ & $\mathbf{2 4 3}$ & $\mathbf{0 . 6 5}$ & $\mathbf{0 . 7 5}$ & $\mathbf{0 . 7 1 2}$ \\
*CAUD048 & $\mathbf{2 7}$ & $\mathbf{2 4 1}$ & $\mathbf{0 . 6 7 6}$ & $\mathbf{0 . 8 7 2}$ & $\mathbf{0 . 8 5 7}$ \\
Overall & $\mathbf{9 . 3 8}$ & & $\mathbf{0 . 4 9 2}$ & $\mathbf{0 . 6 2 3}$ & $\mathbf{0 . 5 8 4}$ \\
\hline E & & &
\end{tabular}

$\mathrm{k}$, the number of alleles; N, number of calculated samples; Ho, observed heterozygosity value; He, expected heterozygosity value; PIC, polymorphism information contents.

* Selected 14 microsatellite markers for discrimination analysis. and breeds. Botstein et al. (1980) reported that markers with $\mathrm{He}>0.6$ and $\mathrm{PIC}>0.5$ were the most reliable for discrimination. Based on this information, our $18 \mathrm{MS}$ marker combinations, excluding eight markers (AMU52, CAUD009, $011,127,128$, and 132) could have enough discriminating power to distinguish among individuals and breeds. In a previous study, CAUD009 and CAUD011 had 0.00 and 0.57 PIC values, respectively, for Peking ducks (Yinhua et al., 2005). The results were similar to those of our study in that PIC values of the two markers were 0.207 and 0.492 , respectively, in Southeast Asian ducks. Interestingly, the CAUD009 marker exhibited higher variation in our study than in the Pecking duck study, which suggested the Asian duck population might have more variation than the Pecking duck population. Moreover, Yinhua et al. (2005) reported five alleles and 0.30 for PIC in the CAUD005 of Peking duck populations, but an Asian duck population exhibited nine alleles and 0.728 for PIC, which suggested that the variation in Asian ducks was twice that in Peking ducks. In addition, CAUD031 and CAUD035 had 0.40 and 0.52 PIC values, respectively, and four alleles, but in our study, the PIC values were 0.533 and 0.712 in 10 and 13 number of alleles, respectively, indicating relatively higher variation in Asian duck populations (Yinhua et al., 2005). However, this high variation might have been caused by different sample sizes; the Asian duck population study had 243 samples from eight different populations and the Peking duck population had only 30 samples (Yinhua et al., 2005). Furthermore, the Bangladeshi samples were taken from non-selected random bred populations in which long-term selection for egg or meat production was not practiced, which maintained high heterozygosity in the populations. Liu et al. (2008) also reported on the expected heterozygosity value (He) of $15 \mathrm{MS}$ markers in 26 indigenous duck populations. In their study, CAUD011 (0.714) was 0.141 lower than CAUD066 (0.728) and 0.115 lower than the value for Asian duck populations in which CAUD035 and CAUD044 were calculated similar to HExp values. Alyethodi and Kumar (2010) reported variation for $50 \mathrm{MS}$ markers for the Indian native Moti duck breed, which had lower PIC values than Asian ducks. The same marker, CAUD005, had a PIC value of 0.55, which was 0.178 lower than that of Asian ducks, and interestingly, the CAUD009 marker did not have any alleles in the Moti duck population.

In previous studies, researchers attempted to classify duck breeds using genetic markers for discrimination analysis of domestic duck populations, estimation of birds in seasonal flyways, and pathological purposes, such as discovering the causative agent for AI (Groepper et al., 2014; Ramey et al., 2014). For discrimination analysis using genetic information, genetic markers should include highly polymorphic information, which is needed in various types of genetic markers. Mitochondrial DNA sequence variations 
were frequently used for the identification of wild ducks, and COI gene and D-loop control region were frequently used for duck population discrimination analysis, and also effectively applied to the classification of wild birds (Hebert et al., 2003; Jin et al., 2012; 2014). However, mtDNA sequence variations have limited ability in the discrimination of domestic duck populations, especially Mallards. Thus, MS markers can overcome this problem, have enough discriminating power, and have been widely used for estimation of genetic diversity in animal breed selection (Berthouly et al., 2008).

Although the 18 MS markers have sufficient discrimination power, the $P I$ values were compared in each marker combination to perform efficient and economic analysis. As a result, $14 \mathrm{MS}$ markers were confirmed as the minimum number of markers with high discriminating power for Asian duck populations. Using these marker combinations, $P I$ values were calculated for random, half-sib, and full-sib populations and the values were 1.07E-16, $1.78 \mathrm{E}-12$, and $1.60 \mathrm{E}-06$, respectively. This marker combination is the minimum number of markers for identification of individuals. Because random samples were selected, the probability for appearing to be the same individual was almost zero. This 14-marker combination had an average of 11.67 alleles $(\mathrm{N}), 0.757$ for $\mathrm{He}$, and 0.723 for PIC. In the previous MS marker combination studies, more than 0.6 of $\mathrm{He}$ and PIC values had suitable power of discrimination. Based on the current results, this marker combination has $99.99998 \%$ parentage confirmation power. Therefore, our marker combination can be efficiently used for duck discrimination analysis.

\section{Genetic distance and phylogenetic analysis}

Calculation of genetic distance can illustrate the flow of population differentiation using the allele frequencies and is a useful method for population analysis. The 14 MS markers documented in this study contained highly PIC values for discrimination analysis and understanding of population differences. The results of genetic distances among the Asian duck populations indicated lower genetic distance than commercial ducks with an average of 0.214 . The lowest genetic distance of 0.082 occurred between the KND_Y and KND_J populations (Table 2). These results were expected
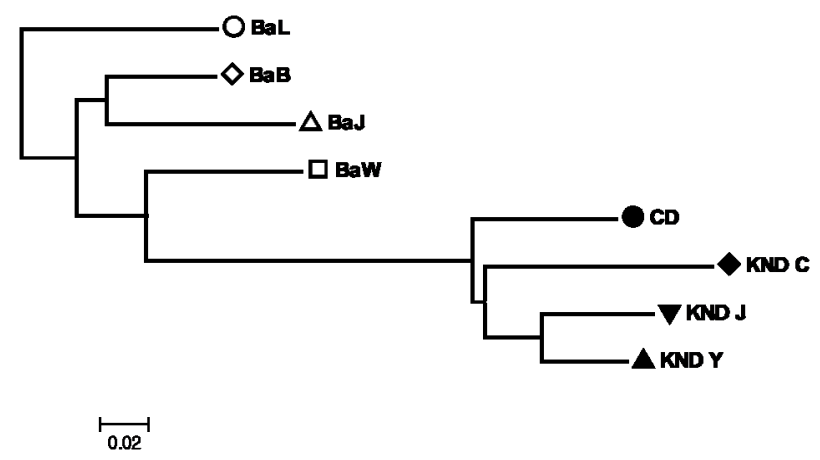

CD, Commercial duck; $\Delta$ KND Yongin; $\boldsymbol{\nabla}$ KND Jangsung; $\bullet$ KND Jangsung colored; $\bigcirc \mathrm{BaL}$, Bangladesh local; $\diamond \mathrm{BaB}$, Bangladesh Black; $\square$ BaW, Bangladesh White; $\triangle$ BaJ, Bangladesh Jinding.

Figure 1. Phylogenetic analysis based on the genetic distances among all populations using 14 selected MS markers. MS, microsatellite, KND, Korean native duck.

because KND_Y and KND_J were the same native Korean duck breed, but were collected from different farms. The largest genetic distance of 0.367 was between the $\mathrm{BaL}$ and KND_C (Table 2). The same result was obtained with phylogenetic analysis between the $\mathrm{BaL}$ and $\mathrm{KND} \_\mathrm{C}$ populations as that derived from genetic distance information (Figure 1). The KND_C population had an average value of 0.337 , which was the longest genetic distance, with the Ba populations (Table 2). Macroscopically, the Ba population was relatively separated from the KND and the commercial duck (CD) populations based on the results from individual phylogenetic analysis (Figure 2). However, KND and CD populations were confirmed to be mixed populations, even though the small group of the CD population was clustered. $\mathrm{Ba}$ and CD populations had an average value of 0.298 , and the $\mathrm{CD}$ population had a relatively close genetic distance with the KND populations with an average distance of 0.138 (Table 2). Phylogenetic analysis using individual samples also indicated that the $\mathrm{CD}$ population was mixed with the KND populations within small-clustered groups.

The Ba populations had an average genetic distance of 0.318 with the KND populations and 0.298 with the CD population, which indicated that the Ba populations were different from $\mathrm{CD}$ and KND (Table 2). Moreover, Ba

Table 2. Genetic distances among the Asian duck populations

\begin{tabular}{|c|c|c|c|c|c|c|c|c|}
\hline Population & $\mathrm{BaB}$ & $\mathrm{BaJ}$ & $\mathrm{BaL}$ & $\mathrm{BaW}$ & CD & KND_C & KND_J & KND_Y \\
\hline $\mathrm{BaB}$ & 0 & & & & & & & \\
\hline $\mathrm{BaJ}$ & 0.124 & 0 & & & & & & \\
\hline $\mathrm{BaL}$ & 0.163 & 0.194 & 0 & & & & & \\
\hline $\mathrm{BaW}$ & 0.145 & 0.205 & 0.184 & 0 & & & & \\
\hline $\mathrm{CD}$ & 0.285 & 0.286 & 0.352 & 0.268 & 0 & & & \\
\hline KND C & 0.329 & 0.364 & 0.367 & 0.287 & 0.164 & 0 & & \\
\hline KND_J & 0.308 & 0.321 & 0.366 & 0.279 & 0.129 & 0.149 & 0 & \\
\hline KND_Y & 0.298 & 0.287 & 0.346 & 0.263 & 0.122 & 0.170 & 0.082 & 0 \\
\hline
\end{tabular}

BaB, Bangladeshi black; BaJ, Bangladeshi Jinding duck; BaL, Bangladeshi Local duck; BaW, Bangladeshi white; CD, commercial duck; KND_C, Jangsung colored Korean native duck; KND_J, Jang-sung white Korean native duck; KND_Y, Yong-in white Korean native duck. 


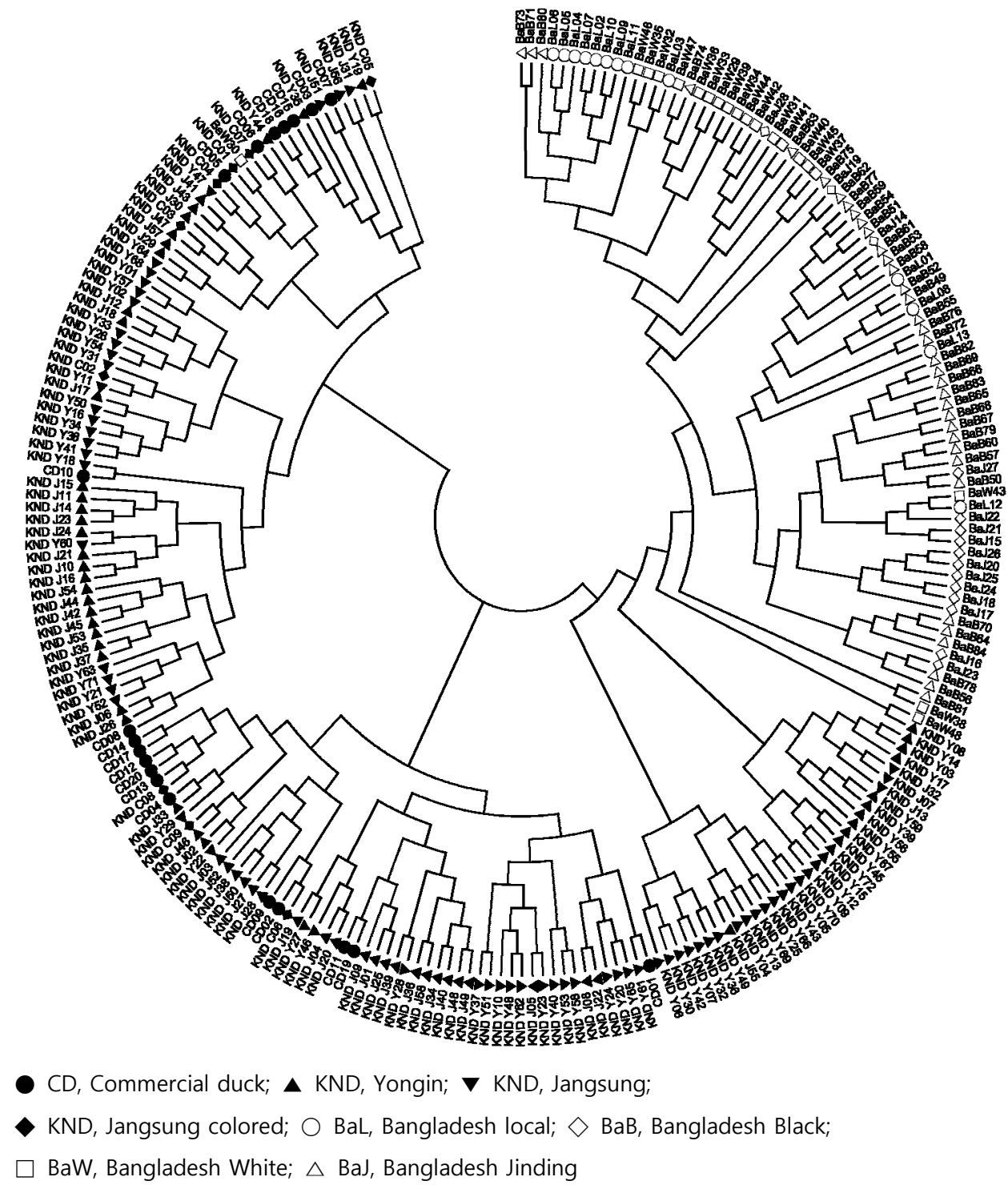

Figure 2. Phylogenetic analysis based on the genetic distances among individual samples using 14 selected microsatellite markers. KND, Korean native duck.

populations were separated as Black $(\mathrm{BaB})$, White $(\mathrm{BaW})$, Jinding (BaJ), and Local (BaL). The average genetic distance among the Ba populations was 0.169 , and was similar to average distance between the $\mathrm{Ba}$ and $\mathrm{KND}$ populations (0.134) (Table 2). Considering these results, the KND and Bangladeshi duck populations were divided into different sub-populations from the respective common ancestor and had separated quite recently (Figure 2).

There are two possible reasons for the close genetic distances among the populations. One reason is that KND and $\mathrm{CD}$ shared the same origin and had alleles from a common ancestor. Based on previous studies, most domestic duck breeds, including Peking ducks, were from the same origin, the Mallard (Omland, 1997). Furthermore, they were near the center of domestic duck origin, South China, which is close to South Korea and Bangladesh. Mallards are one of the frequently moving migratory birds in Asia, and it is possible that gene flow with domestic ducks contributed to its characteristics for ease of domestication. Moreover, interbreeding among different duck varieties is common in Bangladesh and long-term specialized breeding schemes have not yet been adopted in the nucleus flock. Therefore, the resultant gene and genotype frequency did not differ much in Bangladeshi duck varieties. This can also be inferred from the previous discrimination analysis study using the mtDNA COI gene in domestic and wild ducks. The domestic duck (Anas platyrhynchos domesticus) has much closer genetic relationships with the Mallard (0.07) and Spot-billed Duck (0.23) (Jin et al., 2012). Moreover, the highly polymorphic D-loop control region sequence in mtDNA also indicates a very low genetic distance of 0.013 with the Mallard (Jin et al., 2014). Therefore, the mtDNA sequence 
method has limitations for breed discrimination analysis among domestic ducks, Mallards, and Spot-billed Ducks because domestic ducks share alleles and origin from these wild duck species.

Another possibility for increasing the commercial value of native ducks is genetic exchange between the KND and CD populations. Recently, the duck industry has been growing rapidly in Korea because duck meat contains enriched unsaturated fatty acid that consumers recognize as healthy food. Because of the rapid increase in consumer demand, the Korean duck meat industry has developed based on the imported Peking duck, but with lower growth rates and productivity for native Korean ducks. However, recent consumer demand for various types of meat products indicates an increasing preference for native Korean duck. Because of this demand, we suggest crossbreeding the native Korean duck with a commercial duck breed to increase growth rate and productivity. Individual phylogenetic analysis results also estimate the possibility of crossings because the $\mathrm{CD}$ population was clustered in small groups within the KND populations. However, these mixed groups occurred not only because of crossbreeding, but also because the KND population came from domestication of migratory wild ducks in South Asian countries.

\section{Structure analysis}

Genetic structure analysis results helps understanding of haplotype structure. It also confirmed results similar to phylogenetic analysis using genetic distances. When we established a parameter of two for the k-value in KND, CD, and Ba populations, KND and CD populations had the same haplotype structure, whereas the $\mathrm{Ba}$ population had a different haplotype than the other populations (Figure 3). The results were the same with the use of two to eight for k-values; the Ba populations (including four different small groups; W, $\mathrm{B}, \mathrm{L}$, and J) only had one haplotype and was a different population than the KND and CD populations (Figure 3). Moreover, $\mathrm{CD}$ and $\mathrm{KND}$ populations were confirmed to have a mixed haplotype, excluding the Ba populations with three for the k-value (Figure 3). Of interest, the results showed slightly different haplotype patterns in KND_Y and KND_J, although they were the same breed from different farms. These two populations may have different haplotype patterns because of the lack of genetic exchange and independently maintained farms. Additionally, the KND_J population had more similarity with the CD population and we detected similar haplotype patterns from three to eight for k-values, but there were close genetic distances between KND_Y and KND_J (Figure 3).

The $\mathrm{Ba}$ and $\mathrm{CD}$ populations were analyzed for confirmation of haplotype in the Ba populations because the Ba populations were not discriminated among all of the South Asian duck populations. As the result, four of the $\mathrm{Ba}$ populations were clearly separated from the CD population. The other Ba populations have higher similarity, except BaW and part of the $\mathrm{BaB}$ population (Figure not shown). In fact, it

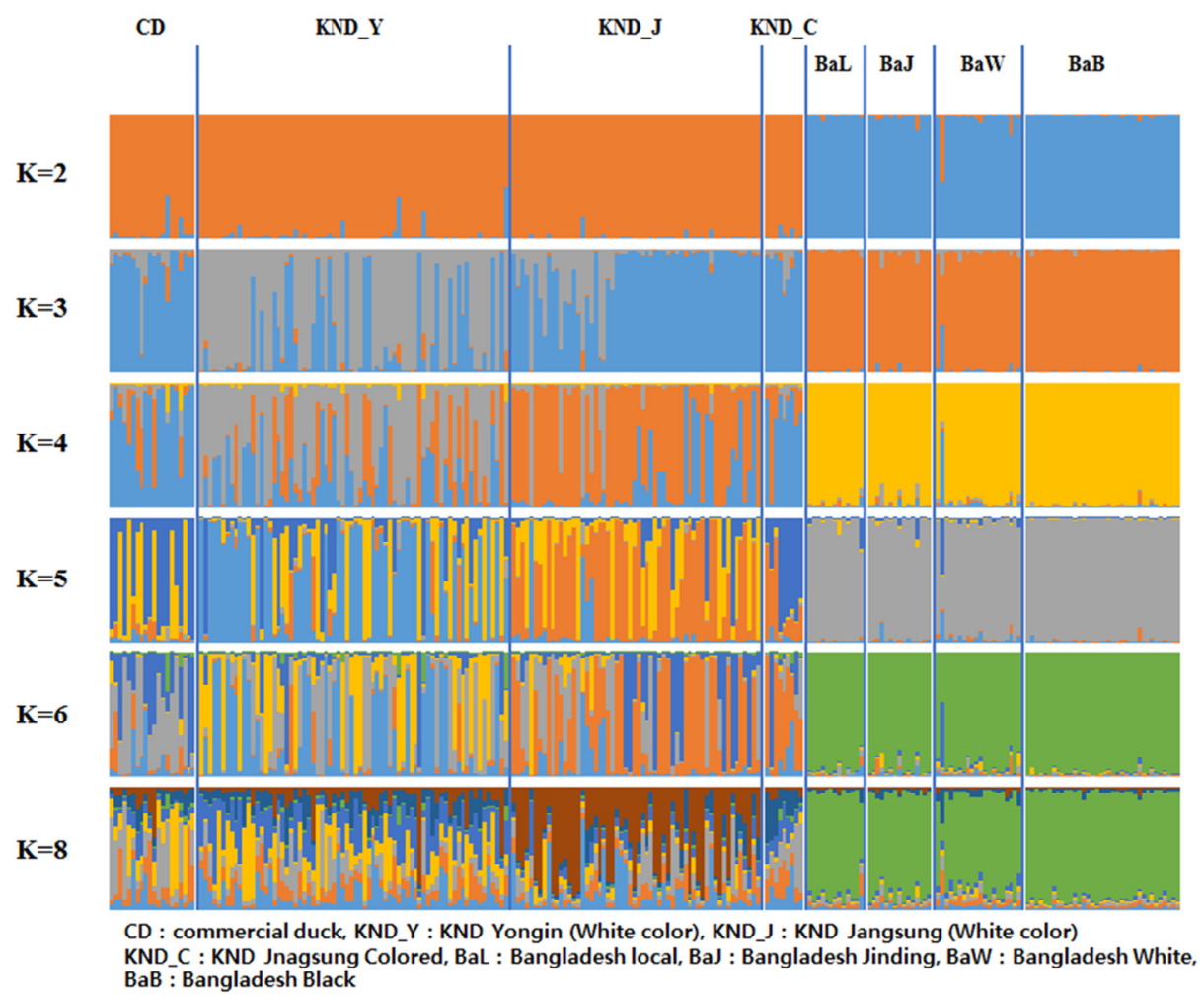

Figure 3. Structure analysis among the Asian duck breeds using 14 selected microsatellite markers. 
was confirmed that the closest genetic distances were among the four groups of the Ba populations and three groups of the KND populations. Ba groups may possibility show differentiation into different lines from one breed.

In this study, we performed genetic distance and phylogeny and structure analysis using MS marker genotypes for discrimination of Asian ducks. Ba populations were fully different from populations of KND and CD, but KND and CD populations were not discriminated well using this MS marker combination. Moreover, Ba populations were well discriminated from other duck populations, but the local native populations were not well discriminated in Bangladesh breeds. However, these 14 MS makers were determined using genotype information to build a database to take advantage of traceability or purebred building strategies because these markers have enough discriminating power for parentage testing and individual discrimination.

\section{CONFLICT OF INTEREST}

We certify that there is no conflict of interest with any financial organization regarding the material discussed in the manuscript.

\section{ACKNOWLEDGMENTS}

This Study was financially supported by research fund of Chungnam National University in 2015.

\section{REFERENCES}

Alyethodi, R. and S. Kumar, 2010. Genetic characterization of moti indian native duck using microsatellite markers. J. Appl. Anim. Res. 38:223-227.

Ayres, K. L. and A. D. J. Overall, 2004. Api-calc 1.0: A computer program for calculating the average probability of identity allowing for substructure, inbreeding and the presence of close relatives. Mol. Ecol. Notes 4:315-318.

Berthouly, C., B. Bed'Hom, M. Tixier-Boichard, C. F. Chen, Y. P. Lee, D. Laloe, H. Legros, E. Verrier, and X. Rognon. 2008. Using molecular markers and multivariate methods to study the genetic diversity of local european and Asian chicken breeds. Anim. Genet. 39:121-129.

Botstein, D., R. L. White, M. Skolnik, and R. W. Davis, 1980. Construction of a genetic linkage map in man using restriction fragment length polymorphisms. Am. J. Hum. Genet. 32:314331.

Dalvit, C., M. De Marchi, and M. Cassandro. 2007. Genetic traceability of livestock products: A review. Meat Sci. 77:437449.

Fulton, J. E. and M. E. Delany. 2003. Poultry genetic resourcesoperation rescue needed. Science 300:1667-1668.

Groenen, M. A. M., R. P. M. A. Crooijmans, A. Veenendaal, H. H. Cheng, M. Siwek, and J. J. van der Poel, 1998. A comprehensive microsatellite linkage map of the chicken genome. Genomics
49:265-274.

Groepper, S. R., T. J. DeLiberto, M. P. Vrtiska, K. Pedersen, S. R. Swafford, and S. E. Hygnstrom, 2014. Avian influenza virus prevalence in migratory waterfowl in the united states, 2007 2009. Avian Dis. 58:531-540.

Hebert, P. D., A. Cywinska, and S. L. Ball. 2003. Biological identifications through DNA barcodes. Proc. R. Soc. London B: Biol. Sci. 270:313-321.

Huang, Y., Y. Zhao, C. S. Haley, S. Hu, J. Hao, C. Wu, and N. Li. 2006. A genetic and cytogenetic map for the duck (Anas platyrhynchos). Genetics 173:287-296.

Jin, S., M. R. Hoque, D. Seo, W. Paek, T. Kang, H. Kim, and J. Lee. 2014. Phylogenetic analysis between domestic and wild duck species in korea using mtdna d-loop sequences. Mol. Biol. Rep. 41:1645-1652.

Jin, S. D., M. R. Hoque, D. W. Seo, I. K. Kim, C. Jo, W. K. Paek, and J. H. Lee. 2012. Phylogenetic relationships among dabbling duck species in Korea using COI gene variations in mtdna. Japan Poult. Sci. 49:163-170.

Kim, H. K., B. S. Kang, J. Hwangbo, C. D. Kim, K. N. Heo, H. J. Choo, D. S. Park, O. S. Seo, and E. H. Hong. 2012. The study on growth performance and carcass yield of meat-type Korean native ducks. Korean J. Poult. Sci. 39:45-52.

Kranis, A., A. A. Gheyas, C. Boschiero, F. Turner, L. Yu, S. Smith, R. Talbot, A. Pirani, F. Brew, P. Kaiser, P. M. Hocking, M. Fife, N. Salmon, J. Fulton, T. M. Strom, G. Haberer, S. Weigend, R. Preisinger, M. Gholami, S. Qanbari, H. Simianer, K. A. Watson, J. A. Woolliams, and D. W. Burt. 2013. Development of a high density $600 \mathrm{k}$ snp genotyping array for chicken. BMC Genomics 14:59.

Kraus, R. H., H. H. Kerstens, P. Van Hooft, R. P. Crooijmans, J. J. Van Der Poel, J. Elmberg, A. Vignal, Y. Huang, N. Li, H. H. Prins, and M. A. Groenen. 2011. Genome wide SNP discovery, analysis and evaluation in mallard (Anas platyrhynchos). BMC Genomics. 12: 150.

Liu, K. J. and S. V. Muse. 2005. Powermarker: An integrated analysis environment for genetic marker analysis. Bioinformatics 21:2128-2129.

Liu, W., Z. C. Hou, L. J. Qu, Y. H. Huang, J. F. Yao, N. Li, and N. Yang. 2008. Population structure and biodiversity of chinese indigenous duck breeds revealed by 15 microsatellite markers. Asian Australas. J. Anim. Sci. 21:314-319.

Maak, S., K. Wimmers, S. Weigend, and K. Neumann. 2003. Isolation and characterization of 18 microsatellites in the peking duck (Anas platyrhynchos) and their application in other waterfowl species. Mol. Ecol. Notes 3:224-227.

Marshall, T. C., J. Slate, L. E. B. Kruuk, and J. M. Pemberton. 1998. Statistical confidence for likelihood-based paternity inference in natural populations. Mol. Ecol. 7: 639-655.

MIFFAF (Ministry for Food, Agriculture, Forestry). 2013. Primary Statistics of Food, Agriculture, Forestry and Fisheries, Sejong, Korea.

Omland, K. E. 1997. Examining two standard assumptions of ancestral reconstructions: Repeated loss of dichromatism in dabbling ducks (Anatini). Evolution 51:1636-1646.

Pritchard, J. K., M. Stephens, and P. Donnelly. 2000. Inference of population structure using multilocus genotype data. Genetics 155:945-959. 
Ramey, A. M., R. L. Poulson, A. S. Gonzalez-Reiche, D. R. Perez, D. E. Stallknecht, and J. D. Brown. 2014. Genomic characterization of H14 subtype influenza a viruses in new world waterfowl and experimental infectivity in mallards (Anas platyrhynchos). Plos One 9:e95620.

Ramos, A. M., R. P. M. A. Crooijmans, N. A. Affara, A. J. Amaral, A. L. Archibald, J. E. Beever, C. Bendixen, C. Churcher, R. Clark, P. Dehais, M. S. Hansen, J. Hedegaard, Z. L. Hu, H. H. Kerstens, A. S. Law, H. J. Megens, D. Milan, D. J. Nonneman, G. A. Rohrer, M. F. Rothschild, T. P. L. Smith, R. D. Schnabel, C. P. Van Tassell, J. F. Taylor, R. T. Wiedmann, L. B. Schook, and M. A. M. Groenen. 2009. Design of a high density snp genotyping assay in the pig using snps identified and characterized by next generation sequencing technology. Plos One. 4:e6524.
Seo, D. W., S. Hasina, N. R. Choi, Y. S. Kim, S. Jin, K. N. Heo, S. D. Jin, and J. H. Lee. 2015. Investigation of microsatellite markers for traceability and individual discrimination of Korean native ducks. Korean J. Poult. Sci. 42:1-8.

Stothard, P., J. W. Choi, U. Basu, J. M. Sumner-Thomson, Y. Meng, X. P. Liao, and S. S. Moore. 2011. Whole genome resequencing of black angus and holstein cattle for SNP and CNV discovery. BMC Genomics 12:559.

Tadano, R., M. Nishibori, N. Nagasaka, and M. Tsudzuki. 2007. Assessing genetic diversity and population structure for commercial chicken lines based on forty microsatellite analyses. Poult. Sci. 86:2301-2308.

Yinhua, H., T. Jianfeng, C. Xuebo, T. Bo, H. Xiaoxiang, L. Zhaoliang, F. Jidong, L. Yankun, L. Li, and X. Ke. 2005. Characterization of 35 novel microsatellite DNA markers from the duck (Anas platyrhynchos) genome and cross-amplification in other birds. Genet. Sel. Evol. 37:455-472. 